\title{
DENOTAMOS O CON SENTIDO O CON REFERENCIA
}

\author{
Rómulo OSCCO LÓPEZ \\ Universidad Ricardo Palma \\ romulo.oscco@urp.edu.pe
}

\section{RESUMEN}

El artículo busca presentar las ideas concernientes a la polémica existente del sentido y la referencia, ya que la presente no busca plantear una alternativa que refiera a tener una delimitación entre la una y la otra, tan solo el propósito de hacer notar que uno de los problemas en el desarrollo de la lógica no solo corresponde a la creación de un nuevo cálculo sin precedentes para el desarrollo de la misma, sino que por el contrario se analizarán otras formas de denotar o hacer referencia en cuanto éstas requieren de una nueva relación semántica.

\section{PALABRAS CLAVE}

Sentido, referencia, denotación, función veritativa.

\section{WE DENOTATE OR WITH SENSE OR WITH REFERENCE}

\begin{abstract}
This article seeks to present ideas relating to the existing polemic of the sense and the reference, since the present does not seek to raise an alternative that recounts to having a delimiting between one and other one, only the intention of making notice that one of the problems in the development of the logic not only corresponds to the creation of a new calculation without precedents for the development of the same one but on the contrary there were analyzed other ways of denoting or referring in all that they need these of a new semantic relation.
\end{abstract}

\section{KEYWORDS}

Sense, reference, denotation, function veritativa.

Recibido: $10 / 4 / 2017$

Aprobado: 15/10/2017 
"Sobre lo que no podemos hablar debemos guardar silencio."

WITTGENSTEIN

$\square$

I comprender a la lógica y sus relaciones inherentes es algo que se ha ido forjando en estos últimos siglos. Desde el desarrollo de las notaciones sintácticas como pretensión de un lenguaje universal como los fallidos lenguajes Volapük o el Esperanto, o del primigenio trabajo exitoso presentado por Frege en su descomunal obra Begriffsschift, Begriffsschrift (Conceptografía). No solo se da un desarrollo a la parte notacional empleándose signos primitivos o construcciones formales, sino también desarrollando una semántica inherente a esta forma de construcción del lenguaje, dándole una importancia a este sentido objetivo el cual debe ser distante de las posturas psicologistas e, incluso, al de los empiristas ingenuos.

Así, el presente trabajo tiene como pretensión sólo el de demarcar o aclarar la relación que existe entre el sentido y la referencia, relación que se convierte en una de las pautas del cambio de concepción frente al psicologismo'. En ese contexto se abordará la temática desde las siguientes perspectivas:

- ¿Cuándo una expresión presenta un sentido?, ¿todo sentido de modo inequívoco plantea una denotación?, ¿cuándo denotamos con una función?

- ¿Qué relación se presenta entre el nombre y la cosa denotada?, ¿qué relación existe entre el nombre y el sujeto y predicado gramatical?, ¿cómo establecemos el valor de verdad o la función veritativa?

Indudablemente, una de las primeras cuestiones es tratar de precisar cómo es que se presenta o plantea el problema del sentido y la referencia ${ }^{2}$, y en ese mismo sentido, el nombre. ${ }^{3}$

No obstante, es necesario tener presente que la idea de la particularidad del nombre surge como una problemática en la concepción de la igualdad entre dos funciones, es decir, si éstas hacen referencia a un nombre o a un objeto o a un signo que se expresa como una imagen de éste:

Sea este el caso:

$$
a=a, a=b
$$

Parece que lo que se quiere decir con $a=b$ es que los signos o nombres « $a$ » $y$ « $b$ »e refieren a lo mismo, y por lo tanto en la igualdad se trataría, precisamente, de estos signos, es decir, se afirmaría una relación entre ellos ${ }^{4}$.

De esto se colige que, de la referencia y el sentido de un signo, es necesario distinguir su representación a él asociada. ${ }^{5}$

La referencia de un nombre propio es el objeto mismo que designamos con él; la representación es totalmente subjetiva ${ }^{6}$.

1 Esta relación es observada por Frege quizás como aquella que no ha permitido el desarrollo de la lógica debido a que no se lograba distinguir el objeto con respecto a la forma de cómo uno se refiere al objeto.

2 En términos de Frege Bedeutung para la referencia y Sinn, para el sentido.

3 Un nombre propio (palabra, signo, fila de signos o expresión) expresa su sentido, se refiere a su referencia o la designa. Con un signo expresamos su sentido y designamos su referencia.

$4 \quad$ Ob. Cit.p 172

5 Ob. Cit. p 176 El ejemplo planteado es el término o expresión Bucéfalo, aquí Frege hace patente la idea de que la diferencia de representación consiste sólo en el modo de darse esta asociación.

6 Debe tenerse presente que el nombre en tanto un proceso mental puede tener un reflejo y sin embargo no puede estar refiriendo a algo en tanto contenido material. 


\section{El actual inca del Perú es diestro.}

Dulce es el nombre de Dulce.

Dulce es dulce.

Sin embargo, en el planteamiento de Frege las expresiones anteriores no pueden ser expresadas en una relación de la forma: "Todo A es B", con lo cual se abre otra necesidad de un cálculo que permita coger estas nuevas relaciones o, en caso contrario, un lenguaje que indique que estas expresiones carecen de referencia.

Podemos ahora distinguir tres niveles de diferenciación entre palabras, expresiones o frases enteras. $O$ bien esta diferencia se refiere a lo sumo a las representaciones; o bien al sentido, pero no a la referencia; o bien, en fin también a la referencia. Sin embargo, es necesario reconocer la pretensión de aquellos que hacen uso del lenguaje, como el caso del poeta al revestir el sentido, debido a que estos matices no son objetivos y solo pretenden llevar al oyente a estas alusiones del lenguaje poético ${ }^{8}$. Sin embargo, debe tenerse presente que estos siempre tienden hacia una representación humana, sin el cual el arte no sería posible.

Un punto a tener presente es que desde ya se pone una limitación a la representación en tanto contenido mental; Frege no asume tal posición en cuanto habla en relación a la designación, ya que esto presupone una referencia.

Ahora indagaremos sobre el enunciado asertivo, entendiendo que un enunciado contiene un pensamiento. El cuestionamiento planteado con ello refiere al hecho de sí es posible el alternar una palabra por otra de la misma referencia, pero de distinto sentido, sin equivocar la referencia de tal enunciado. Sin embargo, se observa que el pensamiento cambia.

Sea el caso de:

$$
\begin{aligned}
& x(x-4) \\
& x^{2}-4 x=x(x-4) \\
& \text { Así } \\
& y=x^{2}-4 x \\
& y=x(x-4) \\
& x^{2}-4 x=x(x-4)^{9}
\end{aligned}
$$
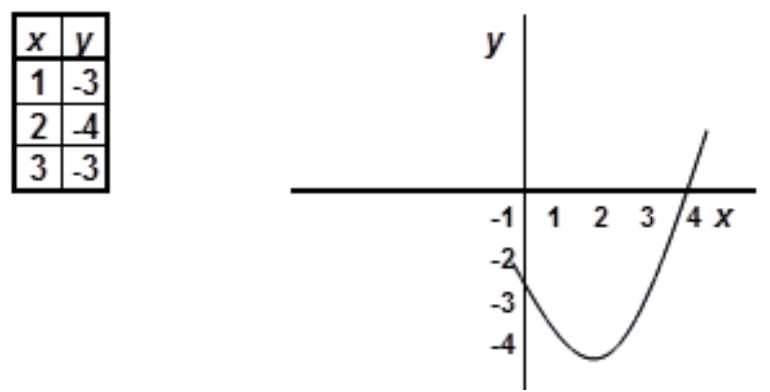

$f=\left\{(x, y) \in R^{2} / y=x^{2}-4 x\right\}$ Donde $: x=1, y=-3 ; x=2, y=-4 ; x=3, y=-3 ; \ldots$

8 Así mismo estas alusiones que se hacen en lenguaje crean una descontextualización por la naturaleza misma del lenguaje.

9 No equiparamos una función con otra, sino solamente el valor de la función con el de la otra. 
Otro caso en relación a esto es el ejemplo planteado por Frege: "El lucero matutino es un cuerpo iluminado por el sol" en relación a: "El lucero vespertino es un cuerpo iluminado por el sol". Es de acuerdo a esto que Frege manifiesta que el pensamiento no puede, pues, ser la referencia del enunciado; por el contrario, sugiere que deberemos de concebirlo como su sentido.

De acuerdo a esto último, es posible conjeturar el hecho de que existen enunciados con sentido pero sin referencia ${ }^{10}$; sin embargo, ¿qué sucede con los enunciados que poseen nombres propios? El actual inca del Perú es diestro, en relación a Heracles fue el mortal que poseía una fuerza divina.

Según Frege, si admitimos la referencia del nombre, entonces estaríamos tentados a darle una referencia a todo el enunciado y con ello a admitirla como verdadera o falsa." Pero, el darle un valor de verdad estaría necesariamente condicionado por la relación de la referencia del predicado. No obstante, hay que reconocer que en tanto el enunciado tenga sentido este sigue siendo un pensamiento. En tanto pensamiento el único interés en ello será el del arte ${ }^{12}$, pues, con ello nos referimos al caso del poeta, por ejemplo.

La búsqueda de la verdad nos motiva para pasar del sentido a la referencia ${ }^{13}$, verdad que se asocia con el de una función veritativa.

Ahora, parece ser necesario tener presente, dónde es que radica este valor veritativo, ya que la articulación del predicado con el sujeto sólo encadena un pensamiento, en tanto que el pensamiento no contiene un valor veritativo por su falta de referencia. En este sentido, un valor veritativo no puede ser parte de un pensamiento, como no puede serlo según el sol ya que este es un objeto y el pensamiento no refiere a objetos, el pensamiento no presenta referencias ${ }^{14}$.

Presentada la relación de la referencia y el sentido, pasamos a mencionar otro nivel del análisis, el cual se realiza en el siguiente sentido. Se asume que si la referencia de un enunciado es su valor veritativo, entonces éste debe permanecer inmodificable cuando una parte del enunciado se sustituye por una expresión de la misma referencia.

Sea el caso:

Miguel Grau murió en el Combate de Angamos.

$$
a=\text { Miguel Grau }
$$

$C x=x$ murió en el Combate de Angamos.

$\mathrm{Ca}$

El Caballero de los Mares ${ }^{15}$ murió en el Combate de Angamos.

$a=$ El Caballero de los Mares

10 Pegaso es un caballo alado, piénsese en este caso. Una condición característica de la proposición es que no solo deba poseer sentido sino que deba designar a una referencia y no representarse la referencia, caso contrario estaríamos en la pretensión de asumir que son las palabras las que contienen verdad..

11 Parece necesario el diferenciar el predicado lógico del predicado gramatical. Efectivamente, sujeto y predicado son parte del pensamiento; para el conocimiento se hallan en el mismo nivel.

12 Es decir, entendiendo que el pensamiento es un estado pleno de subjetividad.

13 Ob. Cit.p 181

14 Pues, este estaría tan solo como un estado mental, parece ser que los estados mentales al no tener una relación concreta con un contenido material suelen ser tan solo actos del pensar.

15 A esta expresión podemos denotarla como una descripción definida, sin embargo debe tenerse presente que a pesar de que sustituye al sujeto lógico del enunciado ella no determina un valor veritativo. 


\section{C $x=x$ murió en el Combate de Angamos. \\ $\mathrm{Ca}$}

Existe al menos alguien que murió en el Combate de Angamos.

$$
(\exists x) M x^{16}
$$

De acuerdo a esto, nunca podemos quedarnos tan sólo con la referencia de un enunciado; pero tampoco el simple pensamiento nos proporciona ningún conocimiento, sino únicamente el pensamiento junto con su referencia, es decir, su valor veritativo. ${ }^{17}$ De ahí que, el juzgar sea el paso de un pensamiento hacia su valor veritativo.

De acuerdo a lo que ya se dijo, el valor veritativo de un enunciado parece ser el de su referencia. Ahora bien, ¿qué pasa si la expresión a sustituirse es un enunciado? Si nuestro análisis es correcto tal como se ha mencionado, el valor veritativo de un enunciado que contiene a otro debe quedar inmodificado si sustituimos el enunciado componente por otro cuyo valor veritativo es el mismo. Es necesario, en este punto, hacer la distinción entre enunciados directos e indirectos; el primero de estos se refiere a otro enunciado; en tanto que el segundo se refiere al pensamiento.

Es necesario tener presente que los gramáticos consideran los enunciados subordinados como representantes de partes del enunciado en general, y, según eso, las denominan enunciados nominales, calificativos y adverbiales. ${ }^{18}$

En nuestro caso la referencia del enunciado subordinado es, en realidad, el pensamiento. Se ve también por el hecho de que, para la verdad del todo, es indiferente que aquel pensamiento sea verdadero o falso.

Sea el caso:

Copérnico creía que las órbitas de los planetas eran circulares.

Copérnico creía que la apariencia del movimiento del Sol es producida por el movimiento de la Tierra.

Aquí el enunciado subordinado así como el enunciado principal tienen como sentido único un solo pensamiento y la verdad del todo no implica ni la verdad ni la falsedad del subordinado. Así, en este caso, no estaría permitido el sustituir una expresión en el enunciado subordinado sino que aquí tendría que ser sustituido por otra expresión que tenga una referencia indirecta; es decir, por el mismo sentido usual. Si alguien quisiera sacar la conclusión: la referencia de un enunciado no es su valor veritativo, ya que posiblemente uno podría estar tentado a sustituir para cada caso el enunciado por otro enunciado con el mismo valor veritativo; habría demostrado en éste, más de lo que el enunciado pretende referir. Por la misma razón podría afirmarse que la referencia de la palabra lucero matutino no es Venus; pues no en todas partes podría decirse Venus en vez de lucero matutino. ${ }^{19}$ Así, sólo puede deducirse que la referencia de un enunciado no siempre es su valor veritativo, y que lucero matutino no siempre se refiere al planeta Venus. A saber, el caso de excepción

\footnotetext{
16 Este último no presenta un valor veritativo por no tener un referente, ya que su campo de relación es ambigua.

17 Ob. Cit.p 181

18 Es manifiesto que las frases nominales con "quien", "que", y las adverbiales con "donde", "cuando", "dondequiera", "cuandoquiera" deben ser interpretadas muchas veces por su sentido como condicionales, por ejemplo "quien toca pintura fresca se ensucia". También las frases adjetivas pueden reemplazar a las condicionales. 
se presenta en los enunciados subordinados que acabamos de examinar, cuya referencia es un pensamiento.

Cuando uno se expresa admitiendo expresiones como: parece que, según Frege, lo que se está tratando de mencionar es me parece que..., opino que.... Lo mismo parece suceder con las expresiones alegrarse, lamentar, aprobar, censurar, esperar, temer. ${ }^{20}$

Así como un convencimiento o una creencia es razón de un sentimiento, también puede ser razón de otro convencimiento, como ocurre en la siguiente inferencia. En el enunciado: De la redondez de la Tierra, Colón infirió que, viajando hacia el oeste, podría alcanzar la India, tenemos como referencia de las partes, dos pensamientos:

- Que la Tierra es redonda,

- Que Colón puede alcanzar la India viajando hacia el oeste.

Aquí lo que importa es que Colón estuviese convencido de las dos partes, y que un convencimiento era la razón del otro. ${ }^{21}$ De acuerdo a esto, el que la Tierra sea realmente redonda y que Colón, viajando hacia el oeste, pudiese realmente alcanzar la India, tal como él pensaba, es indiferente para la verdad de nuestro enunciado; pero no es indiferente que pongamos, en vez de la Tierra, el planeta, que está acompañado de una luna cuyo diámetro es mayor que la cuarta parte de su propio diámetro. También aquí tenemos la referencia indirecta de las palabras. Lo mismo sucede con los adverbiales. ${ }^{22}$ Análogamente ocurre también con las preguntas de la forma dudar de qué, no saber qué..3 Sin embargo, aquí hay que tener cuidado y diferenciarlos de los enunciados adverbiales, en las cuales las palabras tienen su referencia usual. Lingüísticamente, estos casos, se diferencian por el modo del verbo.

En consecuencia, con lo anteriormente mencionado, ahora se analizará a los enunciados subordinados en las que las palabras poseen una referencia usual, sin embargo no presentan un pensamiento con sentido, ni un valor veritativo como referencia.

\section{Sea el caso:}

El que descubrió la forma elíptica de las órbitas planetarias murió en la miseria.

La objeción inmediata a este enunciado sería el siguiente; si este subordinado tuviese un sentido, podríamos expresarlo también como un enunciado principal. Pero esto no es posible debido a que el sujeto gramatical el que no tiene ningún sentido independiente, sino que proporciona las relaciones con el segundo miembro del enunciado, murió en la miseria. De ahí que el sentido del enunciado subordinado no sea un pensamiento completo y que su referencia no sea un valor veritativo, sino $\mathrm{Kepler}^{24}$. De este modo, por lo general toda vez que reconocemos un nombre en el enunciado estamos tentados a buscar su referencia.

20 Aquí de acuerdo a Frege se expresa esto del siguiente modo: Cuando, hacia el fin de la batalla de Belle-Alliance, Wellington se alegró de que los prusianos vinieran, la razón de su alegría era un convencimiento. Si hubiera estado equivocado, no se habría alegrado menos hasta tanto hubiese durado su ilusión, y antes de adquirir el convencimiento de que venían los prusianos no podía alegrarse de ello, si bien, en realidad, ya se acercaban.

21 Ob. Cit.p 185

22 Los enunciados imperativos adolecen de referencia por ello una orden, un ruego, un petitorio no serían pensamientos, no obstante está al mismo nivel del pensamiento.

23 Estas se expresan mediante «quién», «qué», «dónde», «cuándo», "cómo», «por medio de qué», etc.

24 Es necesario tener presente aquí que si uno supiera con exactitud la referencia general, entonces podríamos determinar la referencia y luego posee un valor veritativo, no obstante en ausencia de este criterio no se tendría ninguna referencia. 
Obsérvese que cuando se afirma:

Kepler murió en la miseria.

Uno está en condición de asumir que el nombre Kepler designa algo, es por el contrario, presuposición de la afirmación.

Ahora bien, resulta que las lenguas tienen el defecto de que en ellas son posibles expresiones que, por su forma gramatical, están destinadas a designar un objeto, pero que, en casos especiales, no consiguen este objetivo suyo, porque esto depende de la verdad de un enunciado.

Supongamos el caso:

Kepler murió en la miseria.

Cuya expresión formal sería: Alguien murió en la miseria.

$\mathrm{Ma}$

$(\exists x) M x$

Indudablemente, la verdad del enunciado determina la condición semántica de su fórmula, sin embargo, el caso contrario no muestra tal certeza.

Existió uno que descubrió la forma elíptica de las órbitas planetarias.

El que la subordina:

El que descubrió la forma elíptica de las órbitas planetarias.

En este caso, se designa realmente un objeto, o bien sólo produce la apariencia de ello, careciendo de hecho de referencia. Y así es como parte de su sentido, el pensamiento de que existió uno que descubrió la forma elíptica de las órbitas planetarias. Si esto fuera correcto, la negación debería ser:

El que descubrió por primera vez la forma elíptica de las órbitas planetarias, no murió en la miseria, o bien no hubo nadie que descubriese la forma elíptica de las órbitas planetarias.

Esto radica, en una imperfección del lenguaje, de la que, por lo demás, tampoco está libre del lenguaje simbólico del análisis.

De acuerdo a lo ya mencionado, Frege realizó una relación al análisis de sus estructuras condicionales.

Si un número es menor que 1 y mayor que 0 , también su cuadrado es menor que 1 y mayor que 0 .

Napoleón, que se dio cuenta del peligro para su flanco derecho, dirigió, él mismo, sus

Esto se descompone: guardias corps contra la posición enemiga.

a- Napoleón se dio cuenta del peligro para su flanco derecho.

b- Napoleón dirigió él mismo sus guardias de corps contra la posición enemiga.

Si ahora el sol ya se ha levantado, entonces el cielo está muy nublado.

Con estos casos se puede hacer patente la idea de que por lo general el enunciado subordinado no tiene por sentido ningún pensamiento, sino únicamente una parte de alguno de ellos y, en consecuencia, no tiene por referencia ningún valor veritativo. La razón de esto es que la subordinada o no tiene una referencia directa, sino subordinada o el sentido de la 
subordinada es un pensamiento o es incompleta debido a un componente indeterminado; de modo que sólo en relación con la principal puede expresar un pensamiento, y entonces, sin perjuicio de la verdad del todo, puede ser sustituida por otro enunciado del mismo valor veritativo, siempre y cuando, no existan impedimentos gramaticales.

Consideremos ahora unas situaciones en las que no podemos sustituir enunciados con el mismo valor veritativo. Pues, justamente el enunciado expresa más de lo que expresa por sí solo.

Sea el caso:

Rosario se imagina que, por medio de una plegaria, podrá alcanzar una vacante en el próximo examen de admisión.

En este caso se expresan dos pensamientos, de los cuales, no pertenecen el uno al enunciado principal y el otro al subordinado, a saber:

a- Rosario cree que, por medio de una plegaria, podrá alcanzar una vacante en el próximo examen de admisión.

b- Por medio de una plegaria no podrá alcanzar una vacante en el próximo examen de admisión.

En la expresión del primer pensamiento, las palabras de la subordinada tienen su referencia indirecta, mientras que esas mismas palabras, en la expresión del segundo pensamiento, tienen su referencia usual. Vemos que, en nuestra estructura enunciativa originaria, la subordinada debe tomarse como doble, con distintas referencias de las cuales una es un pensamiento y la otra un valor veritativo.

En el siguiente enunciado:

Como el hielo es menos denso que el agua, flota en el agua.

Se da:

a- El hielo es menos denso que el agua.

b- Si algo es menos denso que el agua, flota en el agua.

c- El hielo flota en el agua. ${ }^{25}$

En este caso podemos admitir que el tercer enunciado es un producto de la relación mediata de los primeros pensamientos, el cual es imposible de obtener a partir de sólo uno de ellos.

Análogamente,

Si el hierro fuera menos denso que el agua, flotaría en el agua.

Aquí, al igual que en el caso ya mencionado, los dos pensamientos refieren a que el hierro no es menos denso que el agua y de que algo flota en el agua si es menos denso que el agua. No obstante, este es un enunciado falso.

Las consecuencias de esto llevan a suponer a Frege que es difícil el agotar todas las posibilidades dadas mediante el lenguaje; pero, con todo, cree haber logrado en lo esencial, las razones por las que no siempre se puede sustituir una subordinada por otra del mismo

25 Obsérvese que tiene la estructura del MPP, debido a que el tercer pensamiento está contenido en los dos primeros. 
valor veritativo, sin perjuicio de la verdad de la estructura enunciativa entera.

Estas razones son:

- Que la subordinada no se refiere a ningún valor veritativo, al expresar sólo una parte de un pensamiento.

- Que la subordinada se refiere ciertamente a un valor veritativo, pero no se limita a esto, al comprender su sentido, además de un pensamiento, una parte de otro pensamiento.

Esto se presenta:

a- En la referencia indirecta de las palabras.

b- Cuando una parte del enunciado alude sólo indeterminadamente, en vez de ser un nombre propio.

De ahí que, resulta con suficiente probabilidad que los casos en que una subordinada no es sustituible por otra del mismo valor veritativo, no demuestran nada en contra de nuestra idea de que el valor veritativo es la referencia del enunciado, el sentido del cual es un pensamiento.

De lo anterior podemos concluir en relación a Frege lo siguiente:

Si en general encontramos que el valor cognoscitivo de « $a=a » \mathrm{y}$ « $a=b$ » es distinto, esto podría explicarse por el hecho de que, para el valor cognoscitivo, el sentido del enunciado, es decir, el pensamiento expresado en él, no entra menos en consideración que su referencia, es decir, su valor veritativo. Ahora bien, si $a=b$, la referencia de «b» es ciertamente la misma que «a», $y$, por lo tanto, también el valor veritativo de « $a=b$ » es el mismo que el de « $a=a »$. Sin embargo, el sentido de «b» puede ser distinto del sentido de «a», y con ello también será el pensamiento expresado en « $a=b$ » distinto del expresado en « $a=a » ;$ pero, entonces los dos enunciados tampoco tienen el mismo valor cognoscitivo. Si como hemos hecho más arriba, por «juicio» entendemos el paso del pensamiento a su valor veritativo, también diremos entonces que los juicios son distintos.

Sea el caso:

El primer número natural divisible por la unidad y por él mismo es un número par y el primer número natural divisible por la unidad y por él mismo es un número primo.

a- El primer número natural divisible por la unidad y por él mismo.

b- Es un número par.

c- Es un número primo.

Resulta que

« $a=a » \mathrm{y} « a=b »$

«El primer número natural divisible por la unidad y por él mismo = a» y

«El primer número natural divisible por la unidad y por él mismo = b»

De donde

«2=a»y «2=b» Luego, «a=b»

« $a=$ número par»y « $a=$ número primo»

De esto se sigue que los enunciados en tanto presenten la misma referencia así presenten sentidos distintos, serán verdaderos o falsos a pesar de que el juicio que se obtenga sea distinto porque el pensamiento en sí es distinto. 


\section{CONCLUSIÓN}

Con el presente artículo, se pretende mostrar la necesidad de establecer la referencia de los enunciados ya sean estos una composición de signos o que estos tengan una referencia parcial, es decir, solo mental como es el caso de duendes o el Pegaso. Tener presente estas distinciones es importante porque deslinda la dualidad del contenido mental en tanto pensamiento que garantiza o garantizará para algunos una función veritativa, este problema ya observado por Husserl y Frege, es un error, pues los contenidos mentales no poseen referencia y por ende carecen de un valor de verdad. No obstante no es posible el acrisolar el lenguaje pues este cambia su sentido según el contexto, pero no es garantía para darle un valor de verdad a las proposiciones.

Es necesario tener presente que la diferencia entre los términos y los nombres solo se especifican en un contexto determinado, ya que las palabras no necesariamente tienden a tener una referencia pero si un sentido, en tal sentido los nombres sugieren la necesidad de existencia de lo nombrado.

La exigencia de los lenguajes formales en tanto representaciones de ese "algo" lógico exige la necesidad de tener solo referencia en tanto cumple con las reglas propias de su construcción. Sin embargo hay que tener presente que cada uno de estos lenguajes formales o naturales presentan en algún casos sentidos totalmente disimiles.

\section{BIBLIOGRAFÍA}

Alchourrón, C. Edi. (1995). Lógica. Madrid:Trota

Dummet, M. (1981). Frege: Philosophy of Language. Cambridge, Mass: Harvard University Press.

-----(1990). La verdad y otros enigmas. México: F.C.E.

Frege, G. (1996). Escritos Filosóficos. Barcelona: Crítica.

------(1972). Lógica y Semántica. Chile: Ediciones universitarias de Valparaíso.

Kenny, A. (1995) . Introducción a Frege. Madrid: Cátedra.

Kripke, S. A. (2006). Wittgenstein a propósito de reglas y lenguaje privado. Madrid: Tecnos.

Mosterín, J. (2012). Los Lógicos. Madrid: Espasa Calpe S.A.

Valdivia, L. (1989). Introducción a la semántica y ontología de Gottlob Frege. México: UNAM. 